\title{
Strain mapping on gold thin film buckling and silicon blistering
}

P. Goudeau ${ }^{1}$, N. Tamura ${ }^{2}$, G. Parry ${ }^{1}$, J. Colin ${ }^{1}$, C. Coupeau ${ }^{1}$, F. Cleymand ${ }^{3}$, H. Padmore ${ }^{2}$

${ }^{1}$ Laboratoire de Métallurgie Physique, UMR 6630 CNRS, Université de Poitiers, SP2MI, Téléport2, Bd M. et P. Curie, BP 30179, F-86962 Futuroscope Chasseneuil cedex

${ }^{2}$ Advanced Light Source, Lawrence Berkeley National Laboratory, 1 Cyclotron Road, MS 2-400, Berkeley, CA, 94270, USA

${ }^{3}$ Laboratoire de Science et Génie des Surface, Ecole des Mines de Nancy, UMR CNRS 7570, Parc Saurupt, F-54042, Nancy

\begin{abstract}
Stress/Strain fields associated with thin film buckling induced by compressive stresses or blistering due to the presence of gas bubbles underneath single crystal surfaces are difficult to measure owing to the microscale dimensions of these structures. In this work, we show that micro Scanning X-ray diffraction is a well suited technique for mapping the strain/stress tensor of these damaged structures.
\end{abstract}

Keywords: Thin film, residual stresses, delamination, micro X-ray diffraction, strain mapping

\section{INTRODUCTION}

Biaxial compressive residual stresses are usually present in thin films deposited at room temperature by direct ion beam sputtering. The stress magnitude is often very high (larger than the elastic limit of the same material in the bulk state) and thus spontaneous delamination phenomenon such as 1D - wrinkling or 2D - buckling may appear for a critical film thickness (relaxation of the stored elastic energy) when extracting the sample from the deposition chamber [1]. In the same way, thin film adherent to a substrate and placed in an axial compression may also induce straight line wrinkles over a critical applied stress. Combined with Atomic Force Microscopy, this kind of experiment allows for determining the adhesion energy from the evolution under compression of the geometrical parameters which define buckling (width, deflection and film thickness) [2].

Although a lot of theoretical works have been done to develop mechanical models and calculations (elasticity of thin plates, fracture mechanics, finite element and analytical calculations) with the aim to get a better understanding of driving mechanisms giving rise to this phenomenon, only a few experimental works have been done on this subject to support these theoretical results and almost nothing concerning local stress/strain measurement mainly because of the small dimension of the buckling (few tens of micrometer).

An equivalent and interesting problem is related to blistering and splitting of hydrogen implanted silicon [3]. Models have been suggested to understand the growth of micro-cracks and then to evaluate the critical stress at the edge above which breakage occurs. The size of the blisters is typically around $10 \mu \mathrm{m}$ in width and thus strain/stress measurements at such small scales are also difficult. 
In this work, we use micro beam X-ray diffraction (micro-XRD) available on synchrotron radiation sources as a local probe (spatial) for analysing stress/strain fields. Following our previous studies on spontaneous buckling [4], we investigated delamination of gold films and also the local surface curvatures in Silicon (100) wafers due to the presence of implanted $\mathrm{H}_{2}$ bubbles. The X-ray diffraction results are correlated with finite element simulations.

\section{EXPERIMENTAL DETAILS}

Two types of samples have been studied. For delamination studies, gold films were deposited by ion beam sputtering on $630 \mu \mathrm{m}$ thick silicon (100) and LiF single crystal substrates. The gold films thicknesses are 630 and $150 \mathrm{~nm}$ respectively. The metallic film is perfectly adherent to the LiF susbtrate. For blistering, (100) silicon wafers were implanted with $\mathrm{H}_{2}^{+}$molecules at 160 $\mathrm{KeV}\left(80 \mathrm{KeV}\right.$ for $\mathrm{H}^{+}$ions) with a dose of $2.510^{16}$ ions $/ \mathrm{cm}^{2}\left(510^{16}\right.$ for $\left.\mathrm{H}^{+}\right)$. This implantation has been followed by an annealing treatment at $250^{\circ} \mathrm{C}$ for $1 \mathrm{H}$ to obtain bubble formation and thus very localised silicon bending or blistering [3]. The corresponding implanted depth calculated with the TRIM code (Projected range + longitudinal straggling) gives the silicon thickness which is bent by the $\mathrm{H}_{2}$ bubbles. The value according to our experimental conditions is $800 \mathrm{~nm}$. The damage structures have been characterized prior to XRD measurements by optical microscopy to identify precisely markers which would be easily found with the X-ray beam for $x-y$ stage calibration and also by AFM for extracting geometrical parameters of wrinkles and blisters. A $\mathrm{Au} / \mathrm{LiF}$ sample set has been deformed during a compression test and studied in situ by AFM measurements [2]. Wrinkles are clearly visible on the post-mortem sample surface as well as dislocation step on the LiF substrate where gold film has been removed.

Micro Scanning X-ray diffraction experiments have been done at the ALS on beam line 7.3.3. [5]. Monochromatic X-ray beam with a photon energy of $6 \mathrm{keV}$ has been chosen for investigating gold thin film samples while polychromatic x-ray beam (5-14 keV) has been used for experiment calibration (sample to detector distance and CCD detector tilt angles) and strain measurement on silicon blistering. Back reflection mode is operating for the diffraction measurements as well as fluorescence scans which are used for localising the markers on the sample surface. The sample to detector distance is generally rather small (about $35 \mathrm{~mm}$ ) which allows a wide diffraction data integration range in the configuration $45^{\circ} / 90^{\circ}$ respectively for the incident $\mathrm{x}$-ray beam angle and the angular detector position. The diffraction patterns are analysed using specific software developed for laue pattern (white beam).

\section{RESULTS AND DISCUSSION}

Gold films: We performed at first a scan with a small step of 2 micron on a circular blister resulting from the presence of residual compressive stresses of about $-400 \mathrm{MPa}$ in the gold film after deposition on the silicon wafer. The procedure for doing this measurement and the results are given in figure 1. The optical image (fig 1.a) allows us to know precisely the location of the buckles to be scanned. The coordinates are defined using a reference point with is given by a step corner realized during the thin film deposition. The gold fluorescence scan in fig. 1 (b) 
gives the $x-y$ stage coordinates of this reference point. The gold film is textured with diffracting rings showing the maximum intensities at the pole directions $(2 \Theta$ axis in the vertical direction and $\pm X$ axis along the ring on each side of the $2 \Theta$ axis). We extracted from the (220) ring the d-spacing and plotted its evolution during the $x-y$ scan on fig. 1 (d). We clearly see a decrease of this value at the top of the buckle which is slightly correlated with a strain/stress relaxation of the compressive stress present in the adherent region. Let us note that the stress-free (220) dspacing calculated from bulk gold lattice parameter $(0.4078 \mathrm{~nm})$ is $0.1442 \mathrm{~nm}$ and the angle between (111) planes and (220) is $\mathrm{X}=35.3^{\circ}$ (pole direction).
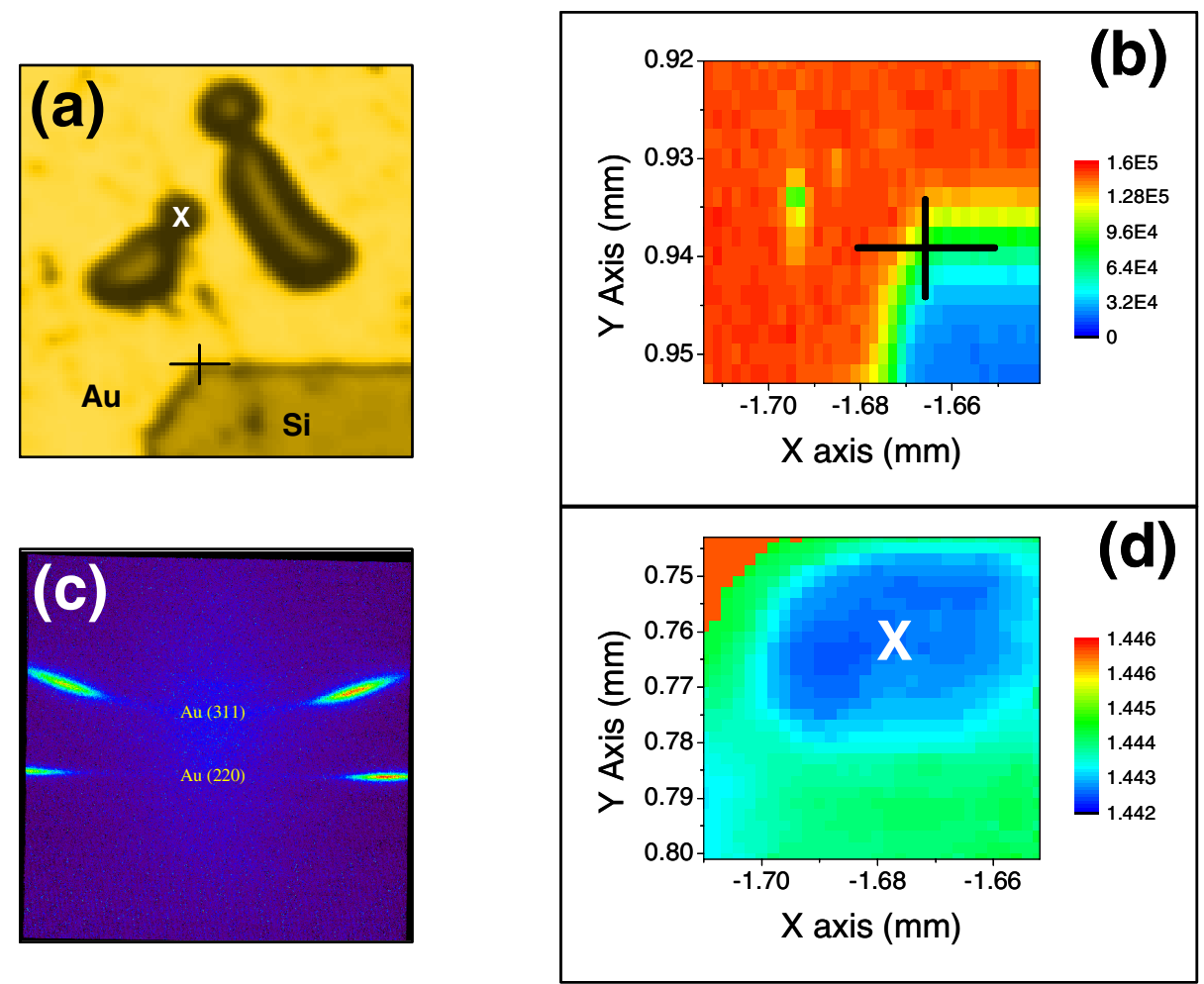

Figure 1: Strain mapping on a gold blister $40 \mu \mathrm{m}$ width. (a) optical image (400 x $400 \mu \mathrm{m})$ of the buckle region and the reference corner marked with the sign + , (b) gold fluorescence scan allowing to obtain the coordinates of the reference point (+), (c) 2D diffraction pattern of the $<111>$ textured gold film and (c) (220) d-spacing variation on the buckle marked with the white cross.

The second example concerns induced delamination after performing an axial compressive test on a LiF single crystal substrate covered with a thin gold film. This work was principally done for studying the effect of metallic film on the emergence process of dislocations nucleated in LiF substrates [2]. The gold film has been partially removed in order to observe by AFM the slip line structures. Straight sized wrinkles appear above a plastic strain of $1.71 \%$ with a deflection and a width estimated to about 0.9 and $9.4 \mu \mathrm{m}$ respectively. The optical image of the sample surface is shown on fig. 2 (a). The reference point for $x-y$ stage calibration corresponds to the white symbol + drawn on the optical image and also the gold fluorescence scan fig. 2 (b). The diffracted intensities in fig 2 (c) are similar but weaker than the one in fig. 1 (c) due to the lower thickness of the film deposited on LiF (ratio 1/4). The variation of the (220) d-spacing 
during the $x-y$ scan is shown fig. 2 (d). The d value is decreasing from the bottom to the top of the wrinkle, in the same way than what is observed for spontaneous buckling fig 1 (d). However, the absolute value of (220) d-spacing is weaker as well as the amplitude of the relaxation. This film was almost stress free after deposition; buckling is created after plastic deformation of $1.7 \%$ which certainly modifies drastically the gold microstructure (elastic domain $<0.3 \%$ ). This effect may then explain the lattice parameter values obtained.
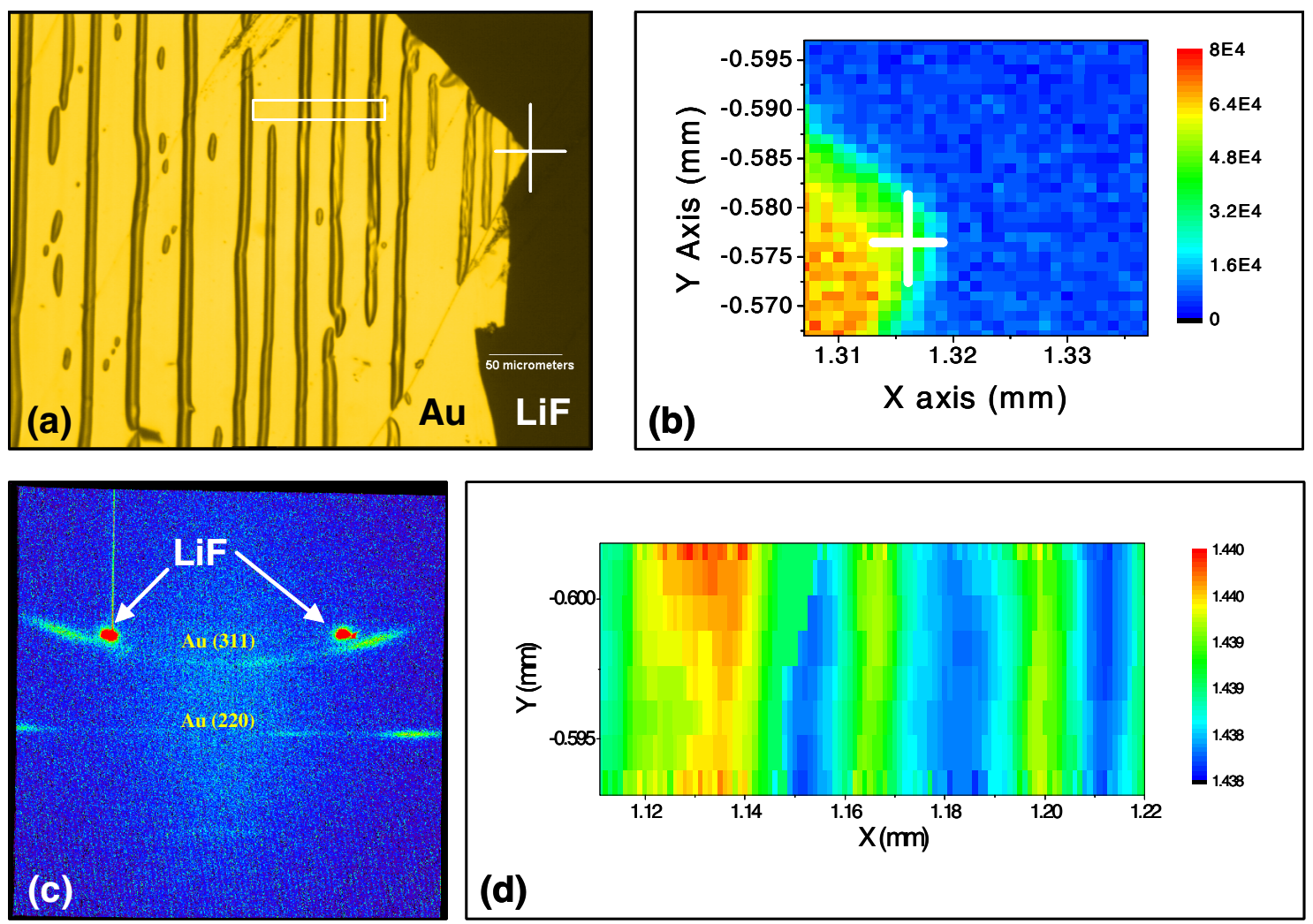

Figure 2: Induced delamination of gold film on LiF substrate: (a) optical image of the gold film surface after $1.7 \%$ plastic deformation; the white rectangle images the zone where XRD scan will be done (b) Gold fluorescence scan of the reference point (c) Diffraction diagram showing the presence of a $<111>$ fibber texture (d) $x-y$ scan performed in the white rectangle of figure (a) with a $1 \mu \mathrm{m}$ step.

Silicon blisters: The main difficulty here is that the entire material is single crystal silicon and thus it appears difficult to differentiate with XRD the bulk wafer signal from the signal due to Silicon blisters. However, XRD is sensitive to crystallographic orientation and then to surface bending. In that preliminary study, we used a $1 \mu \mathrm{m}^{2}$ white beam with a $1 \mu \mathrm{m}$ step to scan the strain field associated with $\mathrm{Si}$ (100) blistering. The geometry of blisters is shown in fig. 3 (a) as well as the breakage effect in (b).

Following an equivalent procedure to the one described above for gold films buckling, XRD measurements have been done over a single blister $9.8 \mu \mathrm{m}$ in width and $135 \mathrm{~nm}$ in height. The Laue pattern obtained when the X-ray beam is at the top of the blister is shown in fig. 4 (a). We clearly observed a ring around the (004) Laue spot of Si bulk structure. In order to get better 
precision, we increased the sample to detector distance from 35 to $235 \mathrm{~mm}$ in order to zoom in on the region close to the (004) Bragg spot. A puzzle or mosaic image of the $x-y$ scan is given on fig 4 (b). The strain analysis of this ring over the Bragg spot is still in progress since specific development software needs to be implemented. However, we can get an idea of the strain variation in this Si blister by using Finite Element simulation. The theory of thin plates can be used since the thickness of the silicon $(0.8 \mu \mathrm{m})$ is large compared to the blister width $(\sim 10 \mu \mathrm{m})$. The results obtained with ABAQUS are given in fig. 5. The mean stress is a maximum at the blister edges (blisters break at the edge - see fig. 3 (b)) and the stress gradients are important (a).

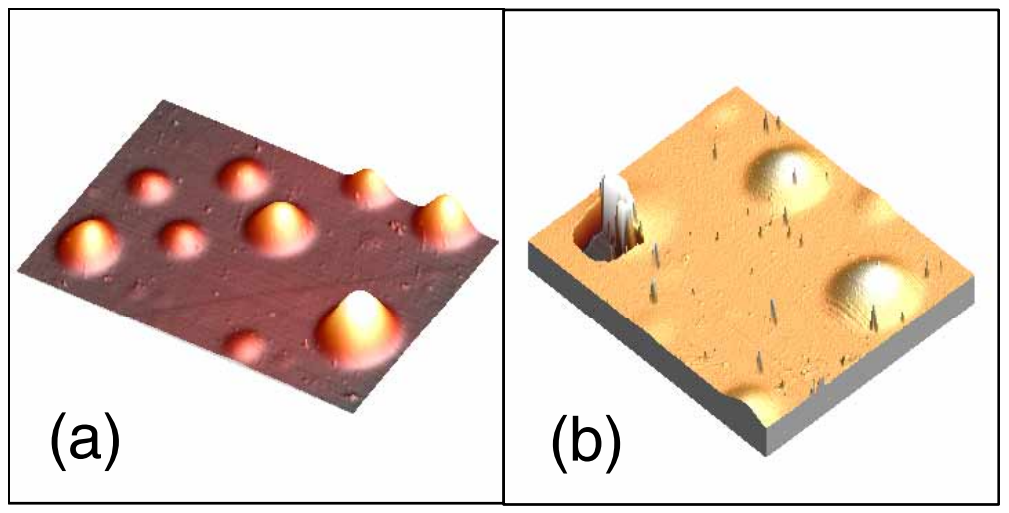

Figure 3: AFM images of the silicon blisters and the effect of breakage in (b) which leads to the presence of small silicon pieces at the sample surface.

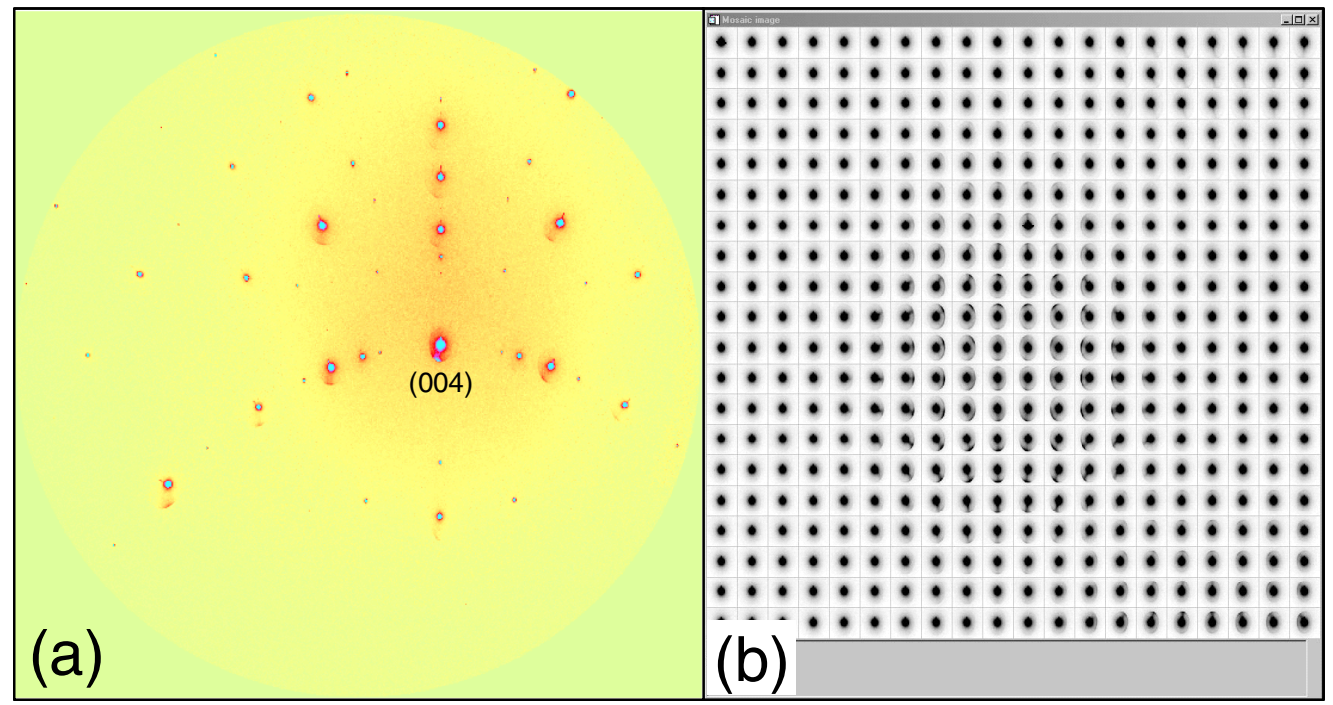

Figure 4: White beam diffraction measurement on the Si blister: (a) diffraction diagram taken at the top of the blister (b) Mozaic image showing the x-y scan performed over the Si blister.

A monochromatic energy scan of (004) Si planes has been done in the energy range of 6318-6733 eV with $5 \mathrm{eV}$ steps, a detector position at $60^{\circ}$, a sample to detector distance at 235 $\mathrm{mm}$, an X-ray beam size larger than the blister width $(\sim 10 \mu \mathrm{m})$, and an exposure time of 60s. The $\mathrm{x}-\mathrm{y}$ stage is fixed at the position at the top of the Si blister. A horizontal curved line is then observed moving from the top to the bottom of the detector with a change in curvature. The 
complete analysis of diffraction patterns needs specific software data treatment for monochromatic wavelength which is in progress.

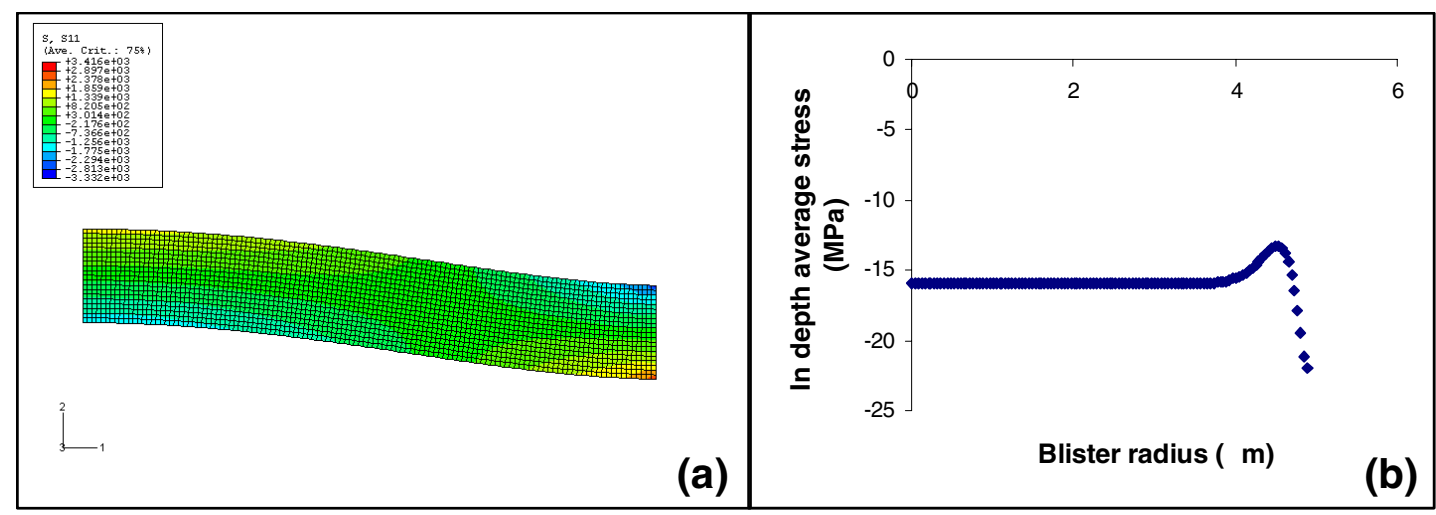

Figure 4: Finite Element simulation of stresses in Silicon blister: (a) cross-section along the radius showing the in depth stress gradient in MPa (b) Mean stress variation along the radius.

\section{CONCLUDING REMARKS}

Micro Scanning X-ray diffraction with either a white or a monochromatic x-ray beam is a powerful technique for exploring microscale damaged structures. Improvements in data treatment are essential for extracting strain/stress tensors from measurements done on Si blisters; software developments are in progress. Furthermore, 3D measurements should be done for extracting in depth stress gradient.

In the case of thin film buckling, the next experimental step will consist in combining compression tests to micro XRD scans for in situ studying the early stage of delamination.

\section{Acknowledgements}

The Advanced Light Source is supported by the Director, Office of Science, Office of Basic Energy Sciences, Materials Sciences Division, of the U.S. Department of Energy under Contract No. DE-AC03-76SF00098 at Lawrence Berkeley National Laboratory. The authors would like to thank Philippe Guerin for thin film deposition and hydrogen implantations in silicon wafers.

\section{REFERENCES}

1. C.Coupeau, P. Goudeau, L. Belliard, M. George, N. Tamura, F. Cleymand, J. Colin, B. Perrin and J. Grilhé, Thin Solid Films 469-470, 221 (2004).

2. C. Coupeau, F. Cleymand and J. Grilhé, Scripta mater. 43, 187 (2000).

3. L.-J. Huang, Q.-Y. Tong, T.-H. Lee, Y.-L. Chao and U. M. Gosele, Electrochemical Society proceedings 19, 1373 (1998).

4. P. Goudeau, P. Villain, N. Tamura, H. Padmore, Applied Physics Letters 83, 51 (2003).

5. N. Tamura, A. A. MacDowell, R. Spolenak, B. C. Valek, J. C. Bravman, W. L. Brown, R. S. Celestre, H. A. Padmore, B.W. Batterman and J. R. Patel, J. Synchrotron Rad. 10, 137 (2003). 